\title{
A Primer on the Physics of the Cosmic Microwave Background
}


This page intentionally left blank 


\title{
A Primer on the Physics of the Cosmic Microwave Background
}

\author{
Massimo Giovannini
}

Centro Enrico Fermi, Italy

CERN, Switzerland 


\section{Published by}

World Scientific Publishing Co. Pte. Ltd.

5 Toh Tuck Link, Singapore 596224

USA office: 27 Warren Street, Suite 401-402, Hackensack, NJ 07601

UK office: 57 Shelton Street, Covent Garden, London WC2H 9HE

\section{British Library Cataloguing-in-Publication Data}

A catalogue record for this book is available from the British Library.

\section{A PRIMER ON THE PHYSICS OF THE COSMIC MICROWAVE BACKGROUND \\ Copyright (C) 2008 by World Scientific Publishing Co. Pte. Ltd. \\ All rights reserved. This book, or parts thereof, may not be reproduced in any form or by any means, electronic or mechanical, including photocopying, recording or any information storage and retrieval system now known or to be invented, without written permission from the Publisher.}

\footnotetext{
For photocopying of material in this volume, please pay a copying fee through the Copyright Clearance Center, Inc., 222 Rosewood Drive, Danvers, MA 01923, USA. In this case permission to photocopy is not required from the publisher.
}

ISBN-13 978-981-279-142-9

ISBN-10 981-279-142-6

Printed in Singapore. 
To Sergio Fubini 
This page intentionally left blank 


\section{Preface}

More than three score years ago, high-energy physicists were driven to scrutinize the properties of the cosmic radiation then available (i.e. cosmic rays). Today the same situation is realized not only with cosmic rays but also with different cosmological data: most notably, with the Cosmic Microwave Background (CMB in what follows). While I am writing this preface, European science is at the forefront of the developments in highenergy physics and cosmology thanks to the Large Hadron Collider program and thanks also to the Planck explorer mission. Today laboratory physics and celestial physics give us contradictory indications: it seems that all the matter accessible to terrestrial laboratory experiments contributes only $5 \%$ to the total energy budget of the Universe.

Cosmologists and astrophysicists today cannot ignore the knowledge of the micro-world provided by high-energy physics. In similar terms, highenergy physicists cannot avoid being exposed to some of the key concepts in modern gravitation and cosmology. While grand unifications of all fundamental forces are one of the intriguing hopes suggested by current theoretical speculations, the opportunity of a small unification lies already before us in the years to come: the construction of a common language which will allow, in the near future, a more effective exchange of information and ideas between contiguous branches of the physics community. The present book seeks to be a modest contribution to this mighty endeavor.

This book grew through the last decade because of various series of lectures that were either directly or indirectly connected to CMB physics and, more generally, to gravitation. In the last couple of years I came to the conclusion that an effective way of presenting a cosmology course (either for last year undergraduate or for $\mathrm{PhD}$ students) is to use $\mathrm{CMB}$ as a guiding theme. While lecturing to $\mathrm{PhD}$ students I have been confronted with the 
problem of giving a sufficiently accurate and updated information to an audience that was, very often, rather composite. Not all $\mathrm{PhD}$ students were exposed to General Relativity or field theory in their undergraduate courses. Similarly, not all PhD students have a preliminary knowledge of astrophysics. I have tried, therefore, to present the material in a reasonably self-contained manner also in view of the time limitations imposed by a $\mathrm{PhD}$ course.

My warm acknowledgment goes to the Enrico Fermi center for a senior research grand entitled From the Planck Scale to the Hubble Radius. Without this support my efforts would have been forlorn. In commencing this script I wish also to express my very special and sincere gratitude to Prof. G. Cocconi and Prof. E. Picasso. I am indebted to G. Cocconi for his advice in the preparation of the first section. I am indebted to E. Picasso for delightful discussions which have been extremely relevant both for the selection of topics and for the overall quality of the manuscript.

Massimo Giovannini 


\section{Contents}

Preface vii

$\begin{array}{ll}\text { Part I } & 1\end{array}$

1. Why CMB Physics? 3

1.1 The blackbody spectrum and its physical implications . 8

1.2 A bit of history of CMB observations . . . . . . . . . . . 12

1.3 The entropy of the CMB and its implications . . . . . . 14

1.4 The time evolution of the CMB temperature . . . . . . . 16

1.5 A quick glance to the Sunyaev-Zeldovich effect . . . . . . 18

1.6 Cosmological parameters . . . . . . . . . . . . 22

2. From CMB to the Standard Cosmological Model 29

2.1 The Standard Cosmological Model (SCM) . . . . . . . 30

2.1.1 Homogeneity and isotropy . . . . . . . . . . . . 31

2.1.2 Perfect barotropic fluids . . . . . . . . . . . 32

2.1.3 General Relativity . . . . . . . . . . . . . 37

2.2 Friedmann-Lemaitre equations . . . . . . . . . . . . . . 40

2.3 Matter content of the SCM . . . . . . . . . . . . . . 44

2.4 The future of the Universe . . . . . . . . . . . . . 47

2.5 The past of the Universe . . . . . . . . . . . 51

2.5.1 Hydrogen recombination . . . . . . . . . . . 54

2.5.2 Coulomb scattering: the baryon-electron fluid . . 59

2.5.3 Thompson scattering: the baryon-photon fluid . 60

2.6 Simplified numerical estimates . . . . . . . . . . . 64 
3. Problems with the SCM 69

3.1 The horizon problem .............. 70

3.2 The spatial curvature problem . . . . . . . . . . . . . 74

3.3 The entropy problem ................. 75

3.4 The structure formation problem . . . . . . . . 76

3.5 The singularity problem ............... 79

4. SCM and Beyond 81

4.1 The horizon and the flatness problems . . . . . . . . . 81

4.2 Classical and quantum fluctuations . . . . . . . . . . . 88

4.3 The entropy problem . . . . . . . . . . . . . . . 93

4.4 The problem of geodesic incompleteness . . . . . . . . 97

5. Essentials of Inflationary Dynamics 101

5.1 Fully inhomogeneous Friedmann-Lemaitre equations . . . 101

5.2 Homogeneous evolution of a scalar field . . . . . . . . . . 108

5.3 Classification(s) of inflationary backgrounds . . . . . . 112

5.4 Exact inflationary backgrounds . . . . . . . . . . . . 116

5.5 Slow-roll dynamics . . . . . . . . . . . . . . 120

5.6 Slow-roll parameters ................ 123

\section{Part III}

6. Inhomogeneities in FRW Models 129

6.1 Decomposition of inhomogeneities in FRW Universes . . 131

6.2 Gauge issues for the scalar modes . . . . . . . . . . . . 133

6.3 Super-adiabatic amplification . . . . . . . . . . 136

6.4 Quantum mechanical description of the tensor modes . . 141

6.5 Spectra of relic gravitons . . . . . . . . . . . . . . . 150

6.6 Quantum state of cosmological perturbations . . . . . . . 151

6.7 Digression on different vacua . . . . . . . . . . . . 156

6.8 Numerical estimates of the mixing coefficients . . . . . . 164

7. The First Lap in CMB Anisotropies 171

7.1 Tensor Sachs-Wolfe effect . . . . . . . . . . . . . . . . 172 
7.2 Scalar Sachs-Wolfe effect . . . . . . . . . . . . . 175

7.3 Scalar modes in the pre-decoupling phase . . . . . . . . 179

7.3.1 Scale crossing and CMB initial conditions . . . . 182

7.4 CDM-radiation system . . . . . . . . . . . . 183

7.5 Adiabatic and non-adiabatic modes: an example . . . . . 188

7.6 Sachs-Wolfe plateau: mixture of initial conditions . . . . 196

8. Improved Fluid Description of Pre-Decoupling Physics 205

8.1 The general plasma with four components . . . . . . 206

8.2 CDM component . . . . . . . . . . . . . 208

8.3 Tight-coupling between photons and baryons . . . . . . 216

8.4 Shear viscosity and silk damping . . . . . . . . . . 218

8.5 The adiabatic solution . . . . . . . . . . . 220

8.6 Pre-equality non-adiabatic initial conditions . . . . . . . 223

8.6.1 The CDM-radiation mode . . . . . . . . . . . . . 224

8.6.2 The baryon-entropy mode . . . . . . . . . . . 228

8.6.3 The neutrino-entropy mode . . . . . . . . . . 229

8.7 Numerics in the tight-coupling approximation . . . . . 232

8.7.1 Interpretation of the numerical results . . . . . 237

8.7.2 Numerical estimates of diffusion damping . . . . 242

9. Kinetic Hierarchies 247

9.1 Collisionless Boltzmann equation . . . . . . . . . . 248

9.2 Boltzmann hierarchy for massless neutrinos . . . . . . 251

9.3 Brightness perturbations of the radiation field . . . . . 255

9.4 Evolution equations for the brightness perturbations . . 257

9.4.1 Visibility function . . . . . . . . . . . . 259

9.5 Line of sight integrals . . . . . . . . . . . . . . 261

9.5.1 Angular power spectrum and observables . . . . 267

9.6 Tight-coupling expansion . . . . . . . . . . . . . 270

9.7 Zeroth order in tight-coupling: acoustic oscillations . . . 272

9.7.1 Solutions of the evolution of monopole and dipole. . . . . . . . . . . . . . . 274

9.7.2 Estimate of the sound horizon at decoupling . . 276

9.8 First order in tight-coupling: polarization . . . . . . . . 278

9.8.1 Improved estimates of polarization . . . . . . . . 278

9.8.2 Polarization power spectra . . . . . . . . . . . 281 
9.9 Second order in tight-coupling: diffusion damping . . . . 287

9.10 Semi-analytical approach to Doppler oscillations . . . . . 292

10. Early Initial Conditions? 305

10.1 Minimally coupled scalar field . . . . . . . . . . . . 307

10.1.1 Gauge-invariant description . . . . . . . . . . 308

10.1.2 Curvature perturbations and scalar normal modes . . . . . . . . . . . . . . . . 310

10.2 Spectral relations . . . . . . . . . . . . . . . 312

10.2.1 Some slow-roll algebra . . . . . . . . . . . . 312

10.2.2 Tensor power spectra . . . . . . . . . . . . 315

10.2.3 Scalar power spectra . . . . . . . . . . . . 318

10.2.4 Consistency relation . . . . . . . . . . . . . 319

10.3 Curvature perturbations and density contrasts . . . . . 320

10.4 Hamiltonians for the scalar problem . . . . . . . . . . . 322

10.5 Trans-Planckian problems? . . . . . . . . . . . . . . 324

10.5.1 Minimization of canonically related

Hamiltonians . . . . . . . . . . . . . . 327

10.5.2 Back-reaction effects . . . . . . . . . . . . 331

10.6 How many adiabatic modes? . . . . . . . . . . . . 334

\section{Part IV}

11. Surfing on the Gauges 341

11.0.1 Generalities on scalar gauge transformations . . 342

11.1 The longitudinal gauge . . . . . . . . . . . . . . 346

11.1.1 Gauge-invariant generalizations . . . . . . . . . 347

11.2 The synchronous gauge . . . . . . . . . . . . . . 349

11.2.1 Evolution equations in the synchronous gauge . . 352

11.2.2 The adiabatic mode in the synchronous gauge. . 357

11.2.3 Entropic modes in the synchronous gauge . . . . 359

11.3 Comoving orthogonal hypersurfaces . . . . . . . . . . 362

11.4 Uniform density hypersurfaces . . . . . . . . . . . . . . 364

11.5 The off-diagonal gauge . . . . . . . . . . . . . 367

11.5.1 Evolution equations in the off-diagonal gauge . . 369

11.6 Mixed gauge-invariant treatments . . . . . . . . . . . . 372 
12. Interacting Fluids 377

12.1 Interacting fluids with bulk viscous stresses . . . . . . . . 379

12.2 Evolution equations for the entropy fluctuations . . . . . 381

12.3 Specific physical limits . . . . . . . . . . . . 385

12.4 Mixing between entropy and curvature perturbations . . 386

13. Spectator Fields 391

13.1 Spectator fields in a fluid background . . . . . . . . . 393

13.2 Unconventional inflationary models . . . . . . . . . . . . 400

13.3 Conventional inflationary models . . . . . . . . . . . 403

Appendix A The Concept of Distance in Cosmology 409

A.1 The proper coordinate distance . . . . . . . . . . . 409

A.2 The redshift . . . . . . . . . . . . . 411

A.3 The distance measure . . . . . . . . . . . . . . . . . . 413

A.4 Angular diameter distance . . . . . . . . . . . . 415

A.5 Luminosity distance . . . . . . . . . . . . . . 416

A.6 Horizon distances . . . . . . . . . . . . . . . . . . . . 417

A.7 Few simple applications . . . . . . . . . . . . . 417

Appendix B Kinetic Description of Hot Plasmas 421

B.1 Generalities on thermodynamic systems . . . . . . . . . 421

B.2 Fermions and bosons . . . . . . . . . . . . . . . . 423

B.3 Thermal, kinetic and chemical equilibrium . . . . . . 425

B.4 An example of primordial plasma . . . . . . . . . . 427

B.5 Electron-positron annihilation and neutrino decoupling . 429

B.6 Big-bang nucleosynthesis $(\mathrm{BBN}) \ldots \ldots \ldots$. . . . . 431

Appendix C Scalar Modes of the Geometry 435

C.1 Fluctuations of the Einstein tensor . . . . . . . . . 435

C.2 Fluctuations of the energy-momentum tensor(s) . . . . 437

C.3 Fluctuations of the covariant conservation equations . . . 439

C.4 Some algebra with the scalar modes . . . . . . . . . . 441

Appendix D Metric Fluctuations: Gauge-Independent Treatment 445

D.1 The scalar problem . . . . . . . . . . . . . . . . . 446

D.2 The vector problem . . . . . . . . . . . . . . 447 
D.3 The tensor problem . . . . . . . . . . . . . . . . . . . . 448

D.4 Inhomogeneities of the sources . . . . . . . . . . . . 449

Bibliography

Index 